\title{
Collaboration in Scientific Visualization
}

\author{
Steve Casera, Peter Kropf \\ Department of Computer Science, University of Neuchâtel, Switzerland \\ steve.casera@unine.ch, peter.kropf@unine.ch
}

\begin{abstract}
This paper discusses the key collaborative features of scientific visualization systems. An experiment with several groups of users has been conducted to evaluate the usability and effectiveness of collaborative tools in such a system. In particular, we compared audio and textual communication support for information exchange as well as the use of graphical indicators. Our analysis revealed the importance of the choice of the conversation means and, surprisingly, the little influence of indicators. Furthermore, the experiments have demonstrated that the system's usability can be significantly improved through appropriate grounding and awareness support. User satisfaction therefore increases, which in turn can potentially also increase the quality of the collaborative work.
\end{abstract}

Keywords: Scientific visualization, CSCW, collaboration.

\section{Introduction}

Visualization is a powerful tool for analyzing data and acquiring knowledge in science, engineering, medicine, finance and many other domains. Work in these areas is most often executed by teams and requires thus support for collaboration. These teams need visualization systems that allow its members, who are often located at geographically distant sites, to jointly investigate the results of a simulation or of an experiment, and to share their knowledge and their experience. The advances in computer and display technologies have largely improved graphical data presentation. Together with the sustained growth of network bandwidth, this has led to the emergence of quite a number of Computer Supported Cooperative Work (CSCW) systems.

Examples of current collaborative visualization systems include AVS with its collaborative extensions [1], CSpray [14], VisAD [10] or gViz [2] (based on Iris Explorer). These systems are generally designed for either optimizing the data transfer or improving the cognitive ease of use, but the majority of them does not pay enough attention to both issues [3].

Comparing these systems is quite difficult, as each is based on different models and has different goals.
Considering the principle performance and collaboration requirements, we have developed a system, called ZoomIn [5], whose objectives include the improvement of the ease of use as well as of the cognitive efficiency and the minimization of the waiting time for the participants in collaborative efforts.

Using ZoomIn, we have conducted a series of experiments to investigate and evaluate the collaborative features of the system. Given that ZoomIn allows to simultaneously present data visualizations to different users at different sites and that it offers them to work on these visualizations individually or coordinated (e.g. guided by a team leader), we analyze in this paper the usefulness and adequacy of the collaborative features of the system. We concentrate on two conversation tools, a textual communication channel (chat) and an audio channel, as well as on two visual indicators, namely avatars and pointers.

Section 2 discusses the principles of grounding and awareness and presents the collaborative features of the ZoomIn visualization tool. The experiment conducted with a group of 24 people, the results obtained and their interpretations are presented in Section 3. We finally conclude in Section 4 by assessing the collaborative features of the visualization system as well as by discussing limitations and possible enhancements.

\section{Collaboration features in ZoomIn}

Real-time collaboration in current distributed groupware workspaces is often a difficult and awkward process. Being aware of what the other participants are doing is an important issue in such collaborations.

\subsection{Grounding}

Participants communicate with each other through exchanging information (messages). The collaboration relies heavily on what is called a common base of knowledge. In particular, the common base of knowledge allows the originator (sender) to make messages believed to be understood by the destination (receiver). The mutual understanding is a condition for the participants in a conversation for using the messages as a contribution to their common knowledge base. Such a collective process by which the partners are trying to expand their common base of knowledge

978 -1-4244-1651-6/08/\$25.00 @ 2008 IEEE 
is called grounding [6]. The collaborative features of ZoomIn for communication and indication, as detailed in the remaining of this section, support the grounding.

\subsection{Workspace awareness}

Awareness may be defined as "knowing what is going on" [7]. This concept of awareness involves states of knowledge as well as dynamic processes of perception and action.

We define workspace awareness as the understanding of the activity of the other person(s) within a shared workspace. Workspace awareness is much harder to maintain in a collaborative environment with distant participants than in a face-to-face situation. It appears often difficult to determine who else is currently active in the workspace, what the other participants are working on, and which actions they currently perform. The principle reasons for these difficulties are that the input and output devices used in CSCW systems generate only a small fraction of the perceptual information that is present in a face-to-face interactions.

\subsection{Support of awareness in ZoomIn}

When working in a collaborative environment, the participants' characteristics and presence must be identified. The representation of the participants in the workspace through visual clues such as color, shape or appearance, supports workspace awareness. Furthermore, the addition of a visual indicator (hand or pointer) improves the expressiveness of a participant's representation.

In ZoomIn, avatars (Figure 1) indicate the location of the participants in the workspace and what they are looking at. Clicking on another user's avatar will switch the view to the partner's current view. A pointer allows to designate particular positions of interest in the visualization workspace; the position of a pointer can easily be positioned and saved for later retrieval. Flags may be put in the world to memorize interesting positions and comments can be added to them.

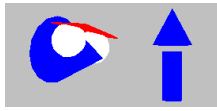

Figure 1. Avatar and pointer in Zoomln

Furthermore, each participant is represented by a color; his/her avatar, pointers and flags are also displayed in that color, so everyone can easily deduce its identity and authorship. Each action executed by participants is indicated by a message; this allows knowing where participants are and which objects they are working on.

Figure 2 shows a screenshot of ZoomIn; the upper right window is the shared world (workspace) with 3D graphical objects, two avatars and a pointer. The middle right window is the private world for individual, not propagated manipulations, i.e. not visible to the collaborators. The lower window serves to send and receive textual information (chat).

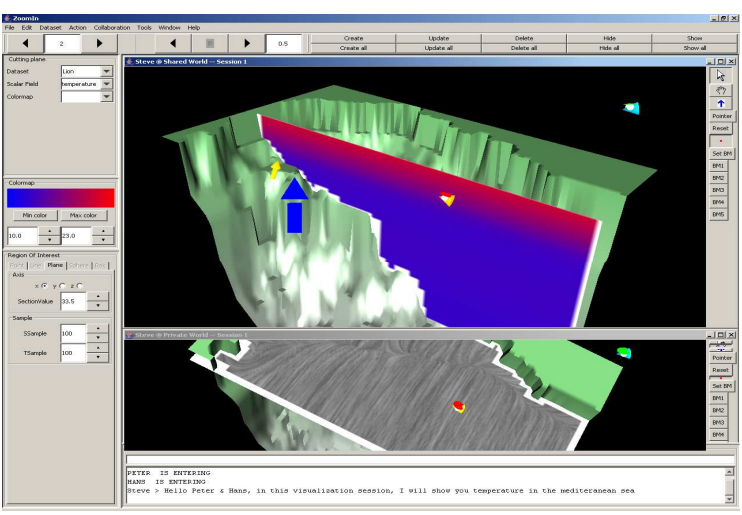

Figure 2. Shared world, private world, and communication channel in Zoomln

\section{Experiment}

In the previous section, we introduced mechanisms to improve the collaboration. However, these features are useless if participants fail to use them adequately. In order to measure its usability, we conducted a simple experiment. In this section, we present the experimentation methods used, state our hypothesis and compare the results obtained against them.

As noted by Neale [12], the evaluation of collaborative systems is much more difficult than evaluating single systems. Individual cognitive factors must be taken into account, as well as collaborative and social factors [13]. Many experiments have been made to evaluate collaborative systems but to our knowledge none for evaluating collaborative scientific visualization systems. We concentrate in this study on the effects of communication media (e.g. as in the experiment by Scholl [15]) and indicators, on their influence on the grounding and awareness, as well as on the usability of the system.

To evaluate the usability of the collaborative features of our system in a situation where people work remotely, we use two sets of comparisons:

1) We compare two media: a textual chat tool (incorporated in the system) and the Skype audio system (combined with the chat tool). For the rest of this paper chat refers to the first case, and audio to the second one.

2) We compare the system with and without using indicators expected to improve awareness. To avoid a long learning phase to the participant, we decided to include only two indicators: avatars and pointers (see Figure 1). For the rest of this paper, the term indicator therefore refers to both avatars and pointers.

Many subjective factors that are difficult to measure are involved in the evaluation of collaborative systems. 
Moreover, only a limited number of skilled participants were available to perform complex and realistic tasks. We therefore asked the participants to just complete a simple task that requires performing elementary actions but which may still be considered as representative enough for scientific visualization. In addition, we add a playful aspect to stimulate the participants to stay engaged in completing the tasks.

\subsection{Experimental conditions}

The task that users have to solve is presented as a game. A pair of participants is facing a 3D world filled with geometric objects showing two basic properties: the shape (cube, sphere, cone or cylinder) and the color (red, pink, green, yellow or brown). In addition, by clicking on the geometric objects, two other properties are displayed: a letter and a reference to the next geometric object (as indicated by its shape and color). The geometric objects are thus arranged in some order.

The goal of the game is to find as fast as possible six geometric objects in the order induced by the references. The six letters obtained this way, placed in the correct order, form a secret code that will open a chest and make the participants winning the game.

In order to enforce collaboration between the two participants, one of them has access to only the color of the next geometric object, while the other participant has access only to its shape. The sharing of these two pieces of information allows to identify the next geometric object.

The shape and color of the initial geometric object are communicated to the participants by the system through a chat message. This is the starting point of the game. The participants have only a single attempt to open the chest; indeed, whether the secret code is correct or not, the game always ends. An example of a 3D world is shown in Figure 3 where the objects are circled for illustration purposes (not visible to the participants). While each task has its own secret code, the $3 \mathrm{D}$ world with its geometric objects is the same for all tasks.

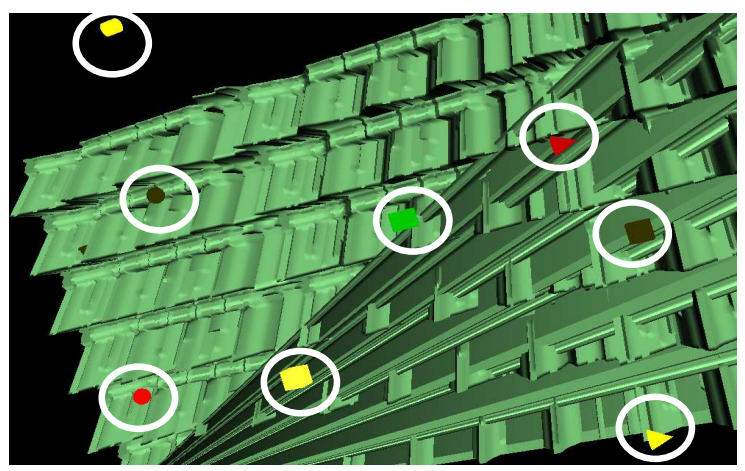

Figure 3. 3D world with geometric objects

The participants perform four games, each consisting of completing a task while using different means of collaboration. The task order is the same for all pairs of participants (in other words, the sequence of secret codes to be discovered is the same for all), only the means vary, as shown in Table 1 . To avoid influencing the statistics, the order of the means used (situations A, $\mathrm{B}, \mathrm{C}$ or D) differs from one pair to another. The actions and conversations of the participants are recorded. This allows determining and analyzing the strategies used, as well as the difficulties encountered by the participants.

Table 1. Means available to complete the task

\begin{tabular}{|c|c|c|}
\hline Mndicator & Chat & $\begin{array}{c}\text { Chat and } \\
\text { audio }\end{array}$ \\
\hline Without avatars \& pointers & situation A & situation C \\
\hline With avatars \& pointers & situation B & situation D \\
\hline
\end{tabular}

24 people participated in pairs in the experiment. They were recruited among the computer science students at our university. Even if more participants would be desirable, we note that this number is comparable to the one in other experiments [8,9] involving between 10 and 20 pairs of participants.

To summarize, the participants must explore the $3 \mathrm{D}$ world, acquire new knowledge and exchange it. We note that the task as such is very elementary and unlikely to occur in scientific visualization. However, participants need to engage in basic interactions and perform actions that are representative of what can be observed in many more realistic situations.

\subsection{Evaluation criteria}

To compare the influence of communication media and indicators, we consider two principle evaluation criteria:

1) Time $t$ to complete the task (effective duration). This is the time between the moment when the participants receive (by chat) the color and shape of the geometric object and the moment when a participant types in the secret code (may it be correct or not).

2) Usability $u$ of the system. This concept is of subjective nature. We thus used a questionnaire (represented in Table 2) that participants had to complete individually after completing a task.

Table 2. Questionnaire evaluating usability components How do you assess the difficulty to complete the task?

\begin{tabular}{|c|}
\hline \\
\hline 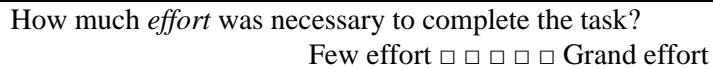 \\
\hline $\begin{array}{l}\text { What level of concentration did you require to complete } \\
\text { the task? }\end{array}$ \\
\hline $\begin{array}{l}\text { How do you assess the difficulty to communicate with your } \\
\text { partner? }\end{array}$ \\
\hline $\begin{array}{l}\text { How difficult was it to move/indicate specific locations in } \\
\text { the world? (awareness) }\end{array}$ \\
\hline $\begin{array}{l}\text { How much time did you take to complete the task? } \\
\text { (perceived duration) } \quad \text { Small } \begin{array}{c}\text { pro } \\
\text { (pig }\end{array}\end{array}$ \\
\hline
\end{tabular}


In the questionnaire, the participant must evaluate, by an integer on a scale of 1 to 5, each of the following components: difficulty, effort, concentration, ease of communication, awareness and perceived duration to complete the task. Each participant completed the questionnaire after each game.

To analyze the effects of using the various communication means we made a number of comparisons involving these means while performing the tasks. For this purpose, we state two hypotheses:

$$
\begin{gathered}
\mathrm{H}_{1}: t_{\text {chat+audio }}<t_{\text {chat }} \text { and } u_{\text {chat+audio }}<u_{\text {chat }} \\
\mathrm{H}_{2}: t_{\text {with indicators }}<t_{\text {without indicators }} \text { and } \\
u_{\text {with indicators }}<u_{\text {without indicators }}
\end{gathered}
$$

For $\mathrm{H}_{1}$, the audio is expected to make communication easier and faster. Indeed, talking is faster than typing a text. With $\mathrm{H}_{2}$, we state that the availability of indicators to reveal the position of the participants and to designate places in the $3 \mathrm{D}$ world should reduce the time necessary to complete the task. These features should also improve usability.

Before starting the experiment, participants attend a presentation explaining the game and the functionalities of the system (moving around in the 3D world, using avatars, pointers, chat, etc.). In addition, a few minutes are given to test the system and to complete the task within a simplified 3D world with only five geometrical objects. Then, each participant is placed in a different room and the first game is launched. At the end of it, each participant fills in the questionnaire. This process is repeated for the other three games. After the fourth game, the participants note their general comments on the experiment.

\subsection{Result}

The effective duration of the games and the answers obtained from the questionnaires have been statistically evaluated. The results were subsequently analyzed considering differences in the variances (ANOVA). As usual, we consider $\mathrm{p}<0.05$ to indicate a significant difference and we include the $F$ values for completeness.

Figure 4 shows the average durations of the games. The 12 pairs of participants need more time to complete the first game than the following ones $(\mathrm{F}=6.51, \mathrm{p}=$ 0,032 with the second game, $F=4.62, p=0,047$ with the third game and $F=10.68, p=0,005$ with the fourth game). In contrast, there was no significant difference (p>0.50) among the second, third and fourth game. This is due to the fact that, in the beginning, the world was unknown, and that the participants did not yet know the system well enough. To ensure that the statistics are not influenced by the order in which situations are presented, each one of the situations A, B, $\mathrm{C}$ and $\mathrm{D}$ of Table 1 is used as often in the first position as the others. The longer duration observed for the first game is thus independent of the situation, but stems entirely from the time necessary to get acquainted with the system and the game itself. One notices that the learning time appears to be restricted to the first game only.

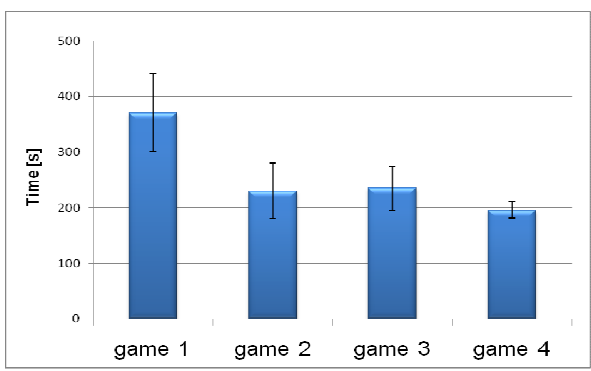

Figure 4. Average duration of the games

To show the effects of the availability of a collaborative mean, we differentiate them (relative to the four situations, see Table 1) for further analysis: without audio (situations $\mathrm{A}$ and $\mathrm{B}$ ), with audio (situations $\mathrm{C}$ and $\mathrm{D}$ ), without indicators (situations $\mathrm{A}$ and $\mathrm{C}$ ), with indicators (situations $\mathrm{B}$ and $\mathrm{D}$ ) as shown in Figure 5 and Figure 6. We recall that chat is always available.

Means:

$\square$ without audio $\square$ with audio $\square$ without indicators $\square$ with indicators

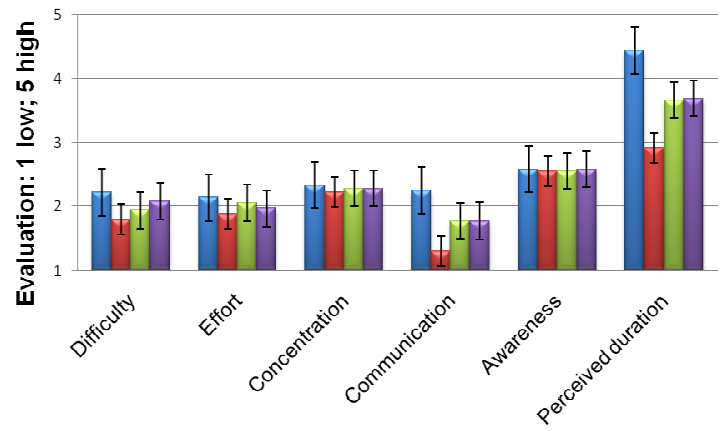

Figure 5. Evaluation of the components of the usability depending on the collaborative feature (mean) available

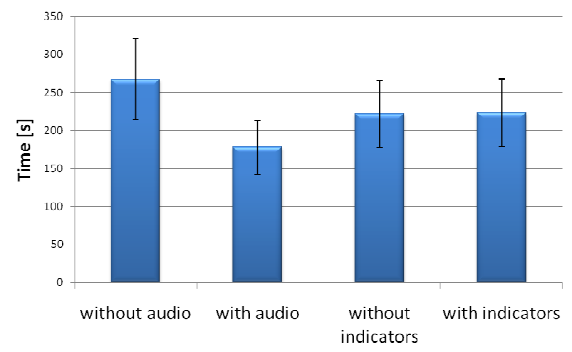

Figure 6. Average effective duration of the games depending on the collaborative feature (mean) available

Table 3 shows the results of the analysis of variance based on the availability of audio. It shows that the use 
of audio has a significant influence on the effective duration $(p=0.0066)$ and on communication $(p<0.001)$. Concerning the difficulty and the perceived duration, the difference is just not significant with a $p$ between 0.05 and 0.06 . Table 4 shows the results of the analysis of variance concerning indicators. The availability of indicators did not significantly affect duration and usability as the value of the smallest $p$ is 0.53 .

Table 3: ANOVA depending of audio

\begin{tabular}{|c|c|c|c|c|c|c|c|}
\hline & $\begin{array}{c}\text { Effective } \\
\text { duration }\end{array}$ & Difficulty & Effort & $\begin{array}{c}\text { Concen } \\
\text { tration }\end{array}$ & $\begin{array}{c}\text { Commu } \\
\text { nication }\end{array}$ & $\begin{array}{c}\text { Aware } \\
\text { ness }\end{array}$ & $\begin{array}{c}\text { Perceived } \\
\text { duration }\end{array}$ \\
\hline$N$ & 24 & 48 & 48 & 48 & 48 & 48 & 48 \\
\hline$F$ & 8.34 & 3.65 & 1.46 & 0.28 & 25.31 & 0.009 & 8.31 \\
\hline$p$ & 0.006 & 0.06 & 0.23 & 0.59 & $3.6 \mathrm{E}-06$ & 0.92 & 0.052 \\
\hline${ }^{\mathrm{c}^{1}}$ & $* *$ & ${ }^{\circ}$ & & & ${ }^{* * *}$ & & ${ }^{\circ}$ \\
\hline
\end{tabular}

Table 4: ANOVA depending of indicators

\begin{tabular}{|c|c|c|c|c|c|c|c|}
\hline & $\begin{array}{c}\text { Effective } \\
\text { duration }\end{array}$ & Difficulty & Effort & $\begin{array}{c}\text { Concent } \\
\text { ration }\end{array}$ & $\begin{array}{c}\text { Communi } \\
\text { cation }\end{array}$ & $\begin{array}{c}\text { Aware } \\
\text { ness }\end{array}$ & $\begin{array}{c}\text { Perceived } \\
\text { duration }\end{array}$ \\
\hline$N$ & 24 & 48 & 48 & 48 & 48 & 48 & 48 \\
\hline$F$ & 0.001 & 0.38 & 0.15 & 0.018 & 0.015 & 0.009 & 0.002 \\
\hline$p$ & 0.95 & 0.53 & 0.69 & 0.91 & 0.89 & 0.92 & 0.96 \\
\hline
\end{tabular}

\subsection{Analysis of the result}

Our first hypothesis $\mathrm{H}_{1}$ states that audio with chat would yield better results than chat only. It turns out that the audio effectively reduces the time needed to complete the task as can be seen in Table 3 and Figure 6. Moreover, the participants found that communication is easier, if they have the audio available as shown in Table 4 and Figure 5. By contrast, the other components of the usability, namely the difficulty, the effort, the concentration, the awareness and the perceived duration are not influenced by the availability of audio. One concludes that the use of audio reduces execution time (effective duration of the game) and thereby improves usability.

As chat is always available with audio, participants also take advantage of the specific features of the chat, such as reviewability (messages can be reviewed later) and revision (message can be reviewed before being transmitted). We note that even if the chat was available with audio, most of the pairs of participants used it only to transmit the letters found and the secret code at the end of the game.

The second hypothesis $\mathrm{H}_{2}$ states that our indicators will reduce the execution time and improve usability. The experiment did not reveal a significant effect with the availability of indicators. Nevertheless, we observed that the participants used avatars to navigate in the $3 \mathrm{D}$ world and pointers to indicate specific locations. This occurred in particular during the first games. For example, if a participant is struggling to find a geometric object, his/her partner will indicate a position close to where this participant should look for. This can be done by using communication media and indicators. The indicators are thus useful under certain circumstances.

The harder the participants attempt to complete a particular task and discover geometric object, the better will be their results. On the other hand, their needs to dispose of an explicit presentation of awareness information will decrease. For example, some participants have used descriptions of positions in commons words: "The pink cube is close to the mountains, near the origin." This is very difficult to achieve at the beginning of the experiment [11], but obviously facilitates the execution of the task. After a few games, a participant knows better the world and becomes more skillful.

The need to use means that contribute to awareness is greater for participants who are not experts in the field or are not familiar with the 3D world in which they work. For example, in scientific visualization, an oceanographer who is familiar with a region, and knows where to look for interesting phenomena would say: "look here, at the mouth of the Rhone." By generalizing this, the importance of awareness is inversely proportional to the degree of knowledge.

\subsection{Analysis of the observations}

By analyzing the actions of the participants and their communication with chat or audio, we note that the participants have implemented various strategies while facing a number of problems.

\section{Detection of misunderstandings}

The letter attached to the geometric object allows the pair of participants to check whether they consider the same object. In case of a misunderstanding, the two participants will check whether they have chosen the correct geometric object. The participant that found the correct object had a tendency to use the avatar and the pointer to indicate the position. This happened quite often and implies a waste of time.

\section{Chat messages}

If participants exchange only important information, typing the text in the chat tool requires little time. Participants tended to use abbreviations to write faster. This phenomenon can be observed in other areas, such as in writing SMS on cell phones. To control that a geometric object considered is the correct one, a particularly effective strategy is to transmit the letter, and to communicate the information (color or shape) that the respective user is the only one to own. So the participants apply the principle of least collaborative effort as described by Clark [6]. 


\section{Grounding}

During a game, participants acquire new knowledge (position of the objects, place to see more objects, etc.). To finish a game as soon as possible they often lack time to share this knowledge. However, just before or just after the end of a game, the participants proceeded to these exchanges in order to have the same common base of information and thus develop a better strategy for the next game. This is a process of grounding.

During the first games, the participants gave a lot of information about the position of objects. With more experience, they tended to share only essential information. For example, the communication of confirmations such as "ok, it was the same letter" were only observed at the beginning of the experiment. This process of grounding (confirming that the participants have the same object) tended to disappear.

\section{Conclusion}

Compared to other systems, we claim that our experiments demonstrated that the visual and audio features of ZoomIn increase its usability and effectiveness with respect to collaboration. One may note that this is also supported by its ease of use and a low waiting time as reported in [4].

Our experiment shows the interest and benefit generated by the audio tool to collaborate. However, we believe that the chat must always be available. Indeed, compared to audio, the chat offers sustained visual information, requires no special equipment and is best suited for users who are not fluent in oral expression in the common language used.

The addition of the two indicators (avatars and pointers) that aim to increase the awareness showed no significant overall effect. Nevertheless, there are situations in which these indicators are useful: this is for example the case when a participant is struggling to find a graphical object.

We observe that the importance of grounding and awareness decreases with the increase of knowledge. We also note that participants use the communication mean that requires the least collaborative effort. We thus believe that, in the worst case, providing awareness information using these indicators will not penalize participants and, in the best case, performance and usability of the system will be improved.

We performed this experiment with pairs of participants. It would be interesting to examine situations with more participants, possibly collaborating in groups with more than two members, and to check whether, in such a situation, the effects of communication media used will be the same (for example, with audio, only one person can speak at a time). In addition, it would be worthwhile to test the contribution of video which would provide information on activities, gestures and emotions of the participants.
More information about ZoomIn can be found at http://iiun.unine.ch/paral/zoomin. The software as well as more documentation can be downloaded.

\section{Acknowledgements}

We would like to thank the participants of the experiments, H. Nägeli for his support of the ZoomIn project and F. Tschan for her advice in designing and analyzing the experiments.

\section{References}

1. AVS, 2007. http://www.avs.com. Last visit: 05.02.2008.

2. Brodlie, K., D. Duce, J. Gallop, M. Sagar, J. Walton, and $\mathrm{J}$. Wood. Visualization in grid computing environments. In Proceedings of IEEE Visualization. 2004. p. 155-162.

3. Brodlie, K., D. Duce, J. Gallop, J. Walton, and J. Wood, Distributed and collaborative visualization. Computer Graphics Forum, 2004. 23(2): p. 223-251.

4. Casera, S., Visualisation scientifique collaborative. $\mathrm{PhD}$ thesis, 2007. University of Neuchâtel, Switzerland.

5. Casera, S., H.-H. Nägeli, and P. Kropf. A collaborative extension of a visualization system. In IEEE Conference on Distributed Frameworks for Multimedia Applications. 2005. Besançon. p. 176-182.

6. Clark, H.H. and S. Brennan, Grounding in communication, In Perspectives on Socially Shared Cognition, L. Resnick, J. Levine, and S. Teasley, Editors. 1991, APA. p. 127-149.

7. Endsley, M., Toward a theory of situation awareness in dynamic systems. Human Factors, 1995. 37(1): p. 32-64.

8. Gutwin, C., M. Roseman, and S. Greenberg. A usability study of awareness widgets in a shared workspace groupware system. In Proceedings of ACM CSCW'96. 1996. Boston. p. 258-267.

9. Hancock, M., J. Miller, S. Greenberg, and S. Carpendale. Exploring visual feedback of change conflict in a distributed $3 D$ environment. In Proceedings of Advanced Visual Interfaces (AVI'06). 2006. Venezia, Italy.

10. Hibbard, W., VisAD: connecting people to computations and people to people. Computer Graphics 1998. 32(3): p. 10-12.

11. Krauss, R.M. and S.R. Fussell. Mutual knowledge and communicative effectiveness. In Intellectual Teamwork: Social and Technical Bases of Collaborative Work. 1990: Erlbaum. p. 111-145.

12. Neale, D., J. Carroll, and M. Rosson. Evaluating computer-supported cooperative work: models and frameworks In Proceedings of the 2004 ACM Conference on CSCW 2004. Chicago, Illinois, USA p. 112-121.

13. Neale, D., D. Dunlap, P. Isenhour, and J.Carroll. Collaborative critical incident development. In Proceedings of the 44th Annual Meeting of the Human Factors and Ergonomics Society. 2000. Santa Monica, CA, USA. p. 598-601.

14. Pang, A. and C. Wittenbrink, Collaborative 3D visualization with CSpray. Computer Graphics, 1997. 17(2): p. 32-41.

15. Scholl, J., J. McCarthy, and R. Harr. A comparison of chat and audio in media rich environments. In CSCW '06: Proceedings of the 2006 20th Conference on Computer Supported Cooperative Work. 2006: ACM. p. 323-332. 